Research Article

\title{
Formalin Fixation as Tissue Preprocessing for Multimodal Optical Spectroscopy Using the Example of Human Brain Tumour Cross Sections
}

\author{
Mona Stefanakis (iD), ${ }^{1,2}$ Anita Lorenz $\left(\mathbb{D},{ }^{1}\right.$ Jörg W. Bartsch $\left(\mathbb{D},{ }^{3}\right.$ Miriam C. Bassler ${ }^{1 D},{ }^{1,2}$ \\ Alexandra Wagner $\left(\mathbb{D},{ }^{1,2}\right.$ Marc Brecht $\mathbb{D}^{1},{ }^{1,2}$ Axel Pagenstecher $\mathbb{D}^{4},{ }^{4}$ Jens Schittenhelm $\left(\mathbb{D},{ }^{5}\right.$

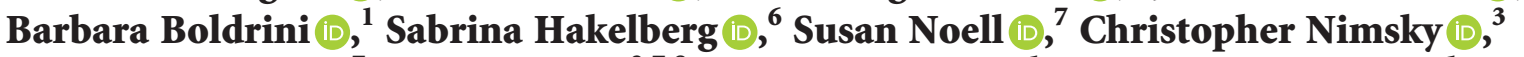 \\ Marcos Tatagiba $\mathbb{D}^{7},{ }^{7}$ Rainer Ritz $\mathbb{D}^{3},{ }^{3,7,8}$ Karsten Rebner $\mathbb{D}^{1},{ }^{1}$ and Edwin Ostertag $\mathbb{D}^{1}$ \\ ${ }^{1}$ Reutlingen University, Process Analysis and Technology PA \& T, Alteburgstraße 150, Reutlingen 72762, Germany \\ ${ }^{2}$ Eberhard Karls University Tübingen, Institute of Physical and Theoretical Chemistry, Auf der Morgenstelle 182, \\ Tübingen 72076, Germany \\ ${ }^{3}$ Department of Neurosurgery, Philipps University Marburg, Baldingerstraße, Marburg 35033, Germany \\ ${ }^{4}$ Department of Neuropathology, Philipps University Marburg, Baldingerstraße, 35033 Marburg, Germany \\ ${ }^{5}$ Institut Für Pathologie und Neuropathologie des Universitätsklinikums Tübingen und Zentrum für Neuroonkologie, \\ Comprehensive Cancer Center Tübingen-Stuttgart, Tübingen University Hospital, Calwerstraße 3, Tübingen 72076, Germany \\ ${ }^{6}$ Deutsche METROHM Prozessanalytik GmbH \& Co. KG, In den Birken 1, Filderstadt 70794, Germany \\ ${ }^{7}$ Department of Neurosurgery, Tübingen University Hospital, Hoppe-Seyler-Straße 3, Tübingen 72076, Germany \\ ${ }^{8}$ Department of Neurosurgery, Schwarzwald-Baar Hospital, Klinikstraße 11, Villingen-Schwenningen 78052, Germany
}

Correspondence should be addressed to Karsten Rebner; karsten.rebner@reutlingen-university.de

Received 18 January 2021; Revised 3 March 2021; Accepted 23 March 2021; Published 13 April 2021

Academic Editor: Feride Severcan

Copyright (c) 2021 Mona Stefanakis et al. This is an open access article distributed under the Creative Commons Attribution License, which permits unrestricted use, distribution, and reproduction in any medium, provided the original work is properly cited.

\footnotetext{
Characterization of brain tumours requires neuropathological expertise and is generally performed by histological evaluation and molecular analysis. One emerging technique to assist pathologists in future tumour diagnostics is multimodal optical spectroscopy. In the current clinical routine, tissue preprocessing with formalin is widely established and suitable for spectroscopic investigations since degradation processes impede the measurement of native tissue. However, formalin fixation results in alterations of the tissue chemistry and morphology for example by protein cross-linking. As optical spectroscopy is sensitive to these variations, we evaluate the effects of formalin fixation on multimodal brain tumour data in this proof-of-concept study. Nonfixed and formalin-fixed cross sections of different common human brain tumours were subjected to analysis of chemical variations using ultraviolet and Fourier-transform infrared microspectroscopy. Morphological changes were assessed by elastic light scattering microspectroscopy in the visible wavelength range. Data were analysed with multivariate data analysis and compared with histopathology. Tissue type classifications deduced by optical spectroscopy are highly comparable and independent from the preparation and the fixation protocol. However, formalin fixation leads to slightly better classification models due to improved stability of the tissue. As a consequence, spectroscopic methods represent an appropriate additional contrast for chemical and morphological information in neuropathological diagnosis and should be investigated to a greater extent. Furthermore, they can be included in the clinical workflow even after formalin fixation.
} 


\section{Introduction}

The digital transformation has made its way into clinical diagnostics and affects the microscopic diagnosis of pathological changes [1]. Digital pathology replaces conventional microscopy since diagnosis can be achieved directly on screen [2]. Further advantages are the combination of data management solutions and evaluation algorithms, as well as the global exchange of data sets $[1,3]$. Investigating a large number of samples in a short time (high throughput screening) requires a digital and predefined process chain, allowing pathologists and digital pathology systems to preselect samples and consequently spend more time with diagnosing [4]. Moreover, the process chain enables a simple integration of additional spectroscopic contrast methods, comparable to classical staining, to improve the selection $[3,4]$. One of these additional contrast methods is optical spectroscopy $[5,6]$. Its implementation into the clinical routine demands a standardized sample handling [7], especially in clinical practice [1, 8]. Therefore, optical spectroscopic methods have to be critically examined for their suitability in clinical routine.

In histopathology, tissue samples are usually obtained from biopsies and resections and immediately treated with formalin for preservation purposes [9]. Formalin is the most used fixative in pathology laboratories [10-13]. This procedure should ensure chemical preservation of the tissue for further investigations and molecular analysis after fixation [10, 11, 14-16]. Although no fixative meets all these criteria, formalin fulfils most of these requirements $[11,17,18]$ as it inhibits autolysis and stabilizes the tissue by cross-linking with amino groups of amino acids present in peptides and proteins. Fixation with formalin does not destroy nucleic acids, carbohydrates, and lipids. In addition, the three-dimensional structure is retained so that it can be explicitly investigated $[9,17,18]$.

After fixation, specimens are commonly embedded in, e.g., paraffin for section preparation, and subsequent tissue sections are histologically stained enabling their microscopic examination [7, 10]. One of the most common routine staining methods is hematoxylin-eosin ( $\mathrm{H} \& \mathrm{E})$ staining. Staining of cell nuclei and cytoplasm allows judgement of cell arrangement, density and number of mitoses, development of necrosis, and nuclear and cellular pleomorphism. These four morphological characteristics are the main criteria for classification of tumours of the central nervous system (CNS) in humans [19]. Based on histopathology and the resulting morphological factors and, more recently, on genetic fingerprints, the World Health Organization (WHO) classifies primary tumours of the CNS in four grades (I-IV) $[19,20]$ with benign tumours classified as WHO grade I. As the highest grade, grade IV indicates the most malignant tumours, such as glioblastoma multiforme [19-22].

Improvement of cancer diagnosis is an ongoing task. Early, targeted and safe cancer therapies can only result in enhanced patient prognosis with a firm basis in diagnosis [23-26]. One approach for a meaningful diagnosis is a combination of microscopic analysis with spectroscopic methods, which does not require any additional preparation effort of the tumour specimen [27, 28]. Therefore, an increasing number of spectroscopic single techniques, especially Raman- and infrared
(IR) spectroscopy, emerge, aiming at the classification of brain tumours [29-32]. Further studies consider elastic light scattering spectroscopy (ELS), which is particularly sensitive to morphological differences in tissues $[33,34]$. It enables one of the earliest detections of carcinogenesis [35-37]. Absorption spectroscopy within the ultraviolet (UV) spectral range is another technique for characterizing cell lines and tumorous tissue [38-40]. Different spectroscopic techniques can complement each other by their information content [41] and thus increase sensitivity and specificity $[42,43]$. This approach is challenged by multimodal optical spectroscopy. It generates large data sets, which are evaluated using multivariate methods to reduce the amount of data and identify small spectral differences [44-46]. In numerous studies, optical spectroscopic techniques in combination with multivariate data analysis methods are used for cancer diagnosis [47-49]. In order to identify and visualize groups within datasets, a principal component analysis (PCA) is an adequate tool [50]. The PCA addresses the extraction of the most relevant information out of a complex data table $[51,52]$. A combination of PCA with the Bayesian discriminant analysis (DA) enables the classification of data and the additional deduction of model-related quality parameters [53]. This PCA-DA-based classification can be subsequently compared with the classical, histopathological diagnosis. Additional quality parameters characterize more precisely the multivariate model and indicate the model's prediction quality.

Up to now, various tissue preparation techniques have been investigated by spectroscopy [54-61]. Most of these studies used IR and Raman spectroscopy for that purpose. It was shown that cells and tissue prepared with formalin are most similar to their native state $[54,58,62]$. However, none of these studies follows a multimodal concept with two or more spectroscopic techniques. In addition, a comparison between spectroscopy-based identification of brain tumours and histopathological tissue sections of these tumours has so far not been performed. In this study, we pursue a multimodal spectroscopic approach using three techniques, UV, ELS, and Fourier-transform (FT) IR spectroscopy. For this purpose, nonfixed and fixed tissue sections of brain tumours are examined with all three spectroscopic methods. We investigate whether formalin fixation is the appropriate fixation protocol for each spectroscopic method. The suitability of formalin fixations to ensure chemical stability is verified with the rarely applied UV and FTIR spectroscopy. Morphological preservation by formalin fixation, however, is investigated with ELS spectroscopy. Based on the multimodal data sets, key performance indicators are calculated for the respective spectroscopic method. Here, we show that multivariate models can be used independently of fixation. Consequently, formalin-fixed tissue sections are suitable for a multivariate model-based diagnosis. Such a diagnostic tool is also simple to implement in a clinical workflow combined with fundamental studies.

\section{Materials and Methods}

2.1. Sample Preparation. Eight human brain tumours of different malignancies and tissue types were excised during surgery and immediately transferred to liquid nitrogen. Two 
different sample sets were used for spectroscopic characterization. Both sets were composed of tumour tissue samples with different WHO grades. Each tumour entity yielded three tissue cross sections. Based on a detailed histopathological evaluation, the most suitable cross section for spectroscopic measurements was chosen.

H\&E staining confirmed that tissues taken for the analyses were derived from the core tumours. Specimens were sectioned at $10 \mu \mathrm{m}$ layer thickness on a cryomicrotome and placed on microscope slides. For UV microspectroscopy, the tissues were prepared on quartz slides (Suprasil 1, Aachener Quarzglas-Technologie Heinrich GmbH \& Co. KG). For the ELS and FTIR microspectroscopy, BioGold slides (SuperChip, Thermo SCIENTIFIC) were used. One half of the slides were subsequently fixed with an aqueous formaldehyde solution (4\% in PBS) and rinsed with PBS, whereas the other half of the slides remained native. Adjacent tissue sections with comparable tissue type and structure were either formalin-fixed or remained native in order to compare the effect of the fixation. Additionally, cross sections of each sample were prepared on glass slides for $\mathrm{H} \& \mathrm{E}$ staining.

2.2. Histopathological Grading. Ten $\mu \mathrm{m}$ thick whole-mount H\&E-stained cross sections from eight tumour tissues were inspected and graded by two independent board-certified neuropathologists (AP and JS). For a detailed list of the samples, see Table S1.

2.3. Data Acquisition. Throughout each tissue cross section, 15 single point measurements were performed for fixed and nonfixed samples of all spectroscopic techniques. Measurement points were selected, considering characteristic morphological features for the different WHO grades. Therefore, tissue heterogeneity was reflected by the measurements.

2.4. Ultraviolet Absorption Microspectroscopy. For each tumour, 15 measurements of each nonfixed and formalin-fixed cross section were acquired. Measurements in transmission were performed on a Zeiss MPM 800 and range from $230 \mathrm{~nm}$ to $380 \mathrm{~nm}$. A blank quartz slide served as reference. Unpolarized light from a XBO-lamp $(14 \mathrm{~V}, 75 \mathrm{~W})$ was spectrally separated by a monochromator (spectral resolution $1 \mathrm{~nm}$, spectral accuracy $\pm 2.5 \mathrm{~nm}$ ) and coupled into the microscope with an illumination aperture of $1.0 \mathrm{~mm}$. The transmitted light was guided through a quartz objective (Zeiss, ULTRAFLUAR 10x, numerical aperture 0.20) and detected by a photomultiplier in the image plane of the microscope. The measuring aperture was $0.25 \mathrm{~mm}$.

2.5. Elastic Light Scattering Microspectroscopy. Fifteen measurements of each nonfixed and formalin-fixed cross sections per tumour were acquired. Measurements in reflection were accomplished on a Zeiss MPM 800 and range from $380 \mathrm{~nm}$ to $700 \mathrm{~nm}$. Unpolarized white light from a halogen lamp $(12 \mathrm{~V}, 100 \mathrm{~W})$ was coupled into a dark-field reflector, and the elastically scattered light was solely collected by the dark-field objective (Zeiss, EPIPLANNEOFLUAR 20x, numerical aperture 0.50). The backscattered light was spectrally separated by the monochromator (spectral resolution $1 \mathrm{~nm}$, spectral accuracy $\pm 2.5 \mathrm{~nm}$ ) and detected by a photomultiplier in the image plane of the microscope. The measured spot was limited to a diameter of $0.63 \mathrm{~mm}$ with an aperture. Spectralon ${ }^{\circledR}$ is used as reference.

2.6. Fourier-Transform Infrared Microspectroscopy. Fifteen measurements of each nonfixed and formalin-fixed tumour cross section were performed in attenuated total reflection with a PerkinElmer Autoimage microscope coupled to a FTIR system 2000 spectrometer. The wavenumbers range from $4000 \mathrm{~cm}^{-1}$ to $700 \mathrm{~cm}^{-1}$. A Germanium crystal with an aperture size of $100 \mu \mathrm{m} \times 100 \mu \mathrm{m}$ was used. The system was referenced against air, and 256 accumulations for each measurement with a gain of 4 were acquired. The spectral resolution was $8 \mathrm{~cm}^{-1}$.

2.7. Data Analysis. The PCA was calculated with the software "The Unscrambler X 10.5" from CAMO with mean centering, leverage correction, and NIPALS-algorithm. Model outliers were identified in the influence plot hotelling's $T^{2}$ versus $F$-residuals (outlier limits 5\% each). Univariate calculations and plots were carried out with OriginPro 2017G from OriginLab Corporation. For the comparison of nonfixed to fixed models, each PCA was combined with a Bayesian discriminant analysis with Mahalanobis distance (UV and ELS microspectroscopy) or Euclidean distance (FTIR microspectroscopy) in CAMO's software "the Unscrambler X 10.5." The number of used principal components (PCs) for the Bayesian discriminant analysis was similar to the shown PCA models. The quality of each model was determined by the degree of accordance between the model's prediction, and the pathologist's assumption was expressed in percent. This value is called overall accuracy. Additionally, the average sensitivity, specificity, false positive rate, and precision were calculated based on the confusion matrix terminology (see explanation of the confusion matrix terminology in the Supplementary Materials for further details) (available here). This results in one model of nonfixed and one model of formalin-fixed samples for each spectroscopic method. Beyond that, an additional model including both the nonfixed and formalinfixed spectra was calculated (Figures S5-S7).

2.8. Spectral Data Processing. The UV spectra were pretreated prior to the multivariate data analysis in the following way: standard normal variate (SNV) and subsequently the Savitzky-Golay $1^{\text {st }}$ (smoothed) derivative (11 points, $2^{\text {nd }}$ polynomial order).

The ELS spectra were preprocessed prior to multivariate data analysis. The initial area normalization was followed by the first SavitzkyGolay (smoothed) derivative (15 points, $2^{\text {nd }}$ polynomial order).

The IR spectra were pretreated prior to multivariate data analysis in the following way: the wavenumbers from 
$2410 \mathrm{~cm}^{-1}$ to $2240 \mathrm{~cm}^{-1}$ were left out due to the $\mathrm{CO}_{2} \mathrm{ab}-$ sorption of air. A unit vector normalization followed by the first SavitzkyGolay (smoothed) derivative (15 points, $2^{\text {nd }}$ polynomial order) was performed.

\section{Results}

3.1. Brain Tumour Samples and Histopathological Grading. In order to validate the diagnosis of the spectroscopically investigated tissue samples, a routine neuropathological diagnosis of the tissue sections was performed by two independent certified neuropathologists (AP and JS). The sample set I consisted of a WHO-grade I fibrous meningioma (in the following mentioned as sample A), a WHO grade II oligodendroglioma (sample B), a WHO grade III anaplastic ependymoma (sample C), and a WHO grade IV glioblastoma (sample D) (Figures 1(a)-1(d)). A second data set was provided to demonstrate the feasibility of this study: sample set II. It consists of a WHO grade I plexus papilloma (in the following mentioned as sample E), a WHO grade II oligodendroglioma (sample F), a WHO grade III anaplastic oligodendroglioma (sample G), and a WHO grade IV glioblastoma (sample H) (Figures 1(e)-1(h)). These tumours were chosen as representative tumour entities for this proofof-concept study (Table S1).

3.2. Ultraviolet Absorption Microspectroscopy of Nonfixed and Formalin-Fixed Human Brain Tumours. Figures 2(a)-2(d) show the UV absorption mean spectra of the human brain tumours. A high absorption at $230 \mathrm{~nm}$ and a broad absorption band between $250 \mathrm{~nm}$ and $300 \mathrm{~nm}$ is visible. The mean spectra have nearly the same shape, and the nonfixed (Figures 2(a) and 2(c)) and formalin-fixed ones (Figures 2(b) and 2(d)) are mostly comparable.

Sample B and sample H, however, reveal small spectral differences between $240 \mathrm{~nm}$ to $260 \mathrm{~nm}$ and $330 \mathrm{~nm}$ to $360 \mathrm{~nm}$ for nonfixed and fixed cross sections. In order to highlight these differences, derivative spectra were generated (Figure S1).

Figures 2(e)-2(l) show the PCA models of the preprocessed spectra and allow a differentiation of the four tumour samples for nonfixed and formalin-fixed cross sections of each sample set. The 3D score plot of the nonfixed cross sections for sample set I is shown in Figure 2(e). On PC1 (79\% explained variance), oligodendroglioma, sample B is separated from the other tissue samples. PC2 (15\% explained variance) demarcates anaplastic ependymoma, sample $\mathrm{C}$ from meningioma, sample $\mathrm{A}$ and glioblastoma, sample D. PC3 (2\% explained variance) distinguishes meningioma, sample A from glioblastoma, sample D. The 3D scores plot of PC1 (86\% explained variance) versus PC2 (7 \% explained variance) versus PC3 (3\% explained variance) of the formalin-fixed brain tumour cross sections for sample set I is shown in Figure 2(f). On PC1, samples A, B, and $\mathrm{C}$ can be distinguished. PC2 and PC3 are needed to demarcate sample D from the other tissue samples. The corresponding loadings plots are shown in Figure 2(i) for the nonfixed model and in Figure 2(j) for the formalin-fixed model and have comparable trends. The main variance of nonfixed and formalin-fixed brain tumour cross sections is located at $240 \mathrm{~nm}$ for PC1. The extrema of the PC2 loadings for the formalin-fixed cross sections shift by $5 \mathrm{~nm}$ to longer wavelengths compared to the nonfixed ones. For the nonfixed cross sections, the extreme values are at $245 \mathrm{~nm}$ and at $280 \mathrm{~nm}$. They are located at $250 \mathrm{~nm}$ and $285 \mathrm{~nm}$ for the formalin-fixed cross sections. On PC3, we observed the main variance at $240 \mathrm{~nm}, 250 \mathrm{~nm}$, and $290 \mathrm{~nm}$ for nonfixed and formalin-fixed cross sections. Comparable PCA models could be achieved with sample set II for the nonfixed (Figures 2(g) and 2(k)) and the formalin-fixed (Figures 2(h) and 2(l)) brain tumour cross sections. Due to the combination of each PCA with a Bayesian discriminant analysis using Mahalanobis distance calculation, a quality comparison of nonfixed and formalin-fixed models was possible. The accordance of the model's prediction with the pathologist's assumption results in $82 \%$ for the nonfixed sample set I and in $85 \%$ the nonfixed sample set II. The formalin-fixed sample set I achieved an overall accuracy of $95 \%$, whereas an accuracy of $75 \%$ was determined for sample set II (Tables S5-S8).

3.3. Elastic Light Scattering Microspectroscopy of Nonfixed and Formalin-Fixed Human Brain Tumours. Figures 3(a)-3(d) show the ELS mean spectra of the human brain tumours for each tissue sample. In each mean spectrum, a broad maximum between $400 \mathrm{~nm}$ and $500 \mathrm{~nm}$ is observable. The spectra are tailing from $500 \mathrm{~nm}$ to $700 \mathrm{~nm}$ and have nearly the same shape. The spectra of nonfixed cross sections (Figures 3(a) and 3(c)) and formalin-fixed ones (Figures 3(b) and 3(d)) are mostly comparable.

Sample C, however, reveals small spectral differences between $400 \mathrm{~nm}$ to $450 \mathrm{~nm}$ for nonfixed and fixed cross sections. In order to highlight these differences, derivative spectra were generated (Figure S3).

Figures 3(e)-3(l) shows the PCA models of the preprocessed spectra. The models allow a differentiation of the tissues for nonfixed sample set I and sample set II and formalin-fixed cross sections of both sample sets. The scores plot of nonfixed cross sections sample set I is shown in Figure 3(e). PC1 (51\% explained variance) distinguishes the tissues. Here, the tissues are located in the orders B, D, C, and A on PC1. PC2 (9\% explained variance) further enhances the separation of the tissues, specifically sample A and sample B from sample C and sample D. The scores plot of PC1 (59\% explained variance) versus PC2 (9\% explained variance) of the formalinfixed brain tumour cross sections sample set $\mathrm{I}$ is shown in Figure 3(f). On PC1, all tissue samples are evenly arranged in the same order as for the nonfixed PCA model. The same applies to PC2, except that PC2 is mirrored. Overall, we find less overlap between the clusters in the scores plot of the formalin-fixed samples. The corresponding loadings plots are shown in Figure 3(i) for the nonfixed model and in Figure 3(j) for the formalin-fixed sample set I model. The main variance of nonfixed and formalin-fixed cross sections is for PC1 at $500 \mathrm{~nm}$. The loadings plots of PC1 have nearly the same shape. For PC2, the main variance and the appearance of the loadings are different. Comparable PCA models could be achieved with sample set II for the nonfixed (Figures $3(\mathrm{~g})$ and $3(\mathrm{k})$ ) and the formalin-fixed (Figures 3(h) and 3(l)) brain 


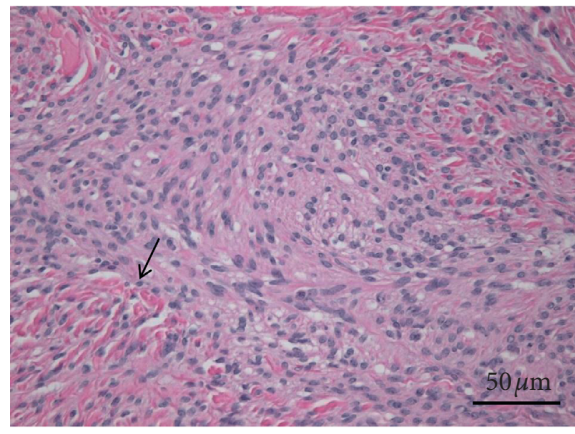

(a)

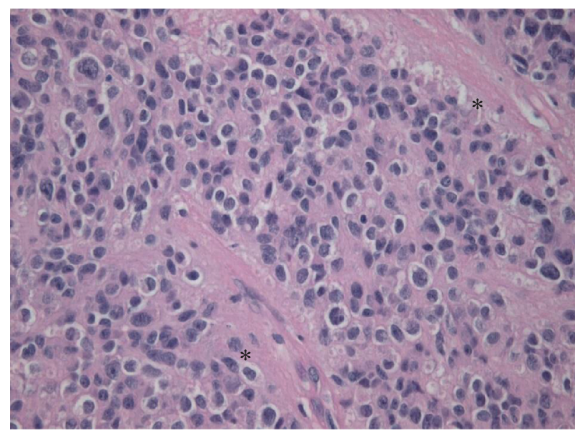

(c)

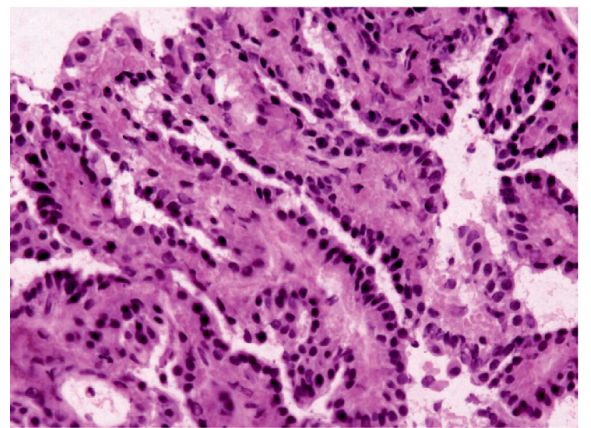

(e)

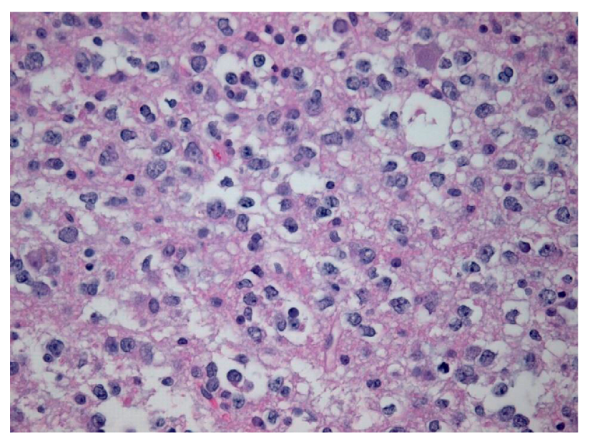

(g)

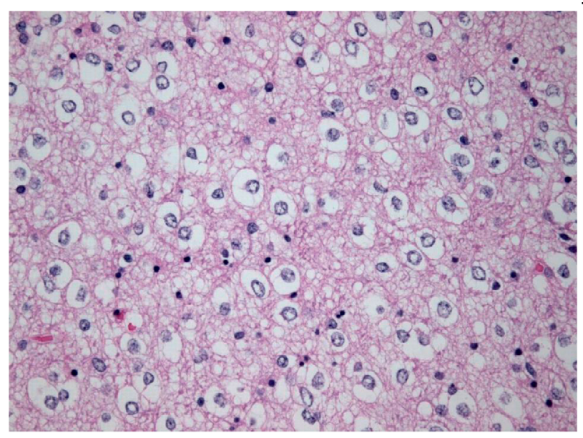

(b)

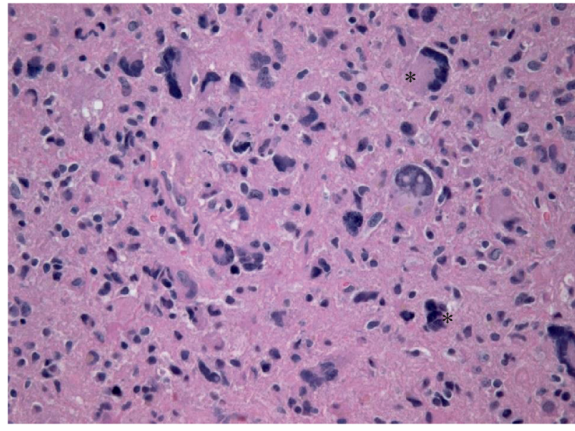

(d)

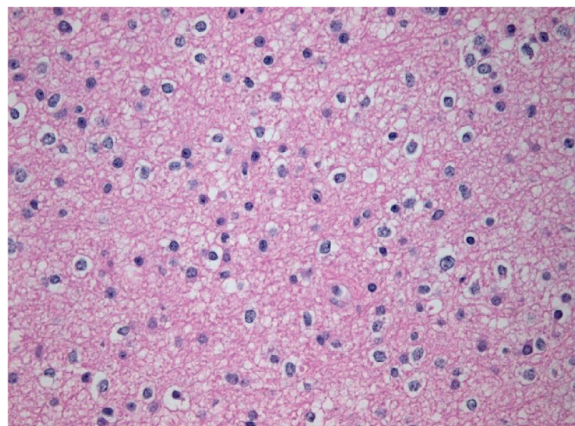

(f)

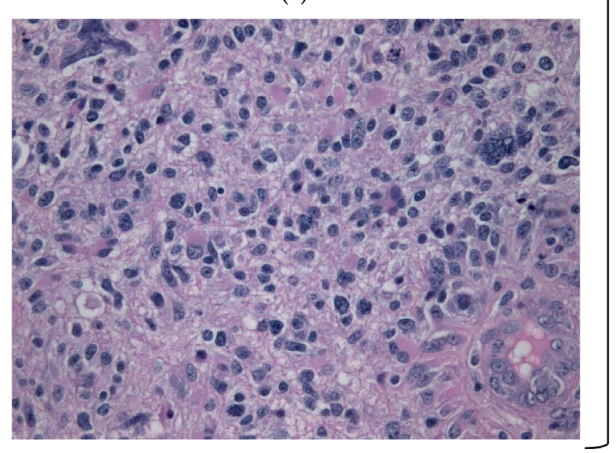

(h)

FIGURE 1: Histological features of brain tumour tissues used for spectroscopic analyses (H\&E staining). (a) fibrous meningioma (WHO grade I sample (A)) with isomorphic tumour cells and eosinophilic collagen fascicles (arrow); (b) low-grade oligodendroglioma (WHO grade II, sample (B)) presented with the typical honeycomb pattern of tumour cells with isomorphic nuclei. Single cells resemble fried eggs with blue yolk (nucleus); neither mitosis nor vascular proliferations are detectable. (c) anaplastic ependymoma (WHO grade III, sample (C)) is a cellular neoplasm with prominent perivascular pseudorosettes (asterisks) and increased proliferation; (d) glioblastoma (WHO grade IV, sample (D)) is characterized by polymorphous tumour cells, some of which are multinucleate (asterisks), by mitotic activity and by prominent vascular proliferation. (e) plexus papilloma (WHO grade I sample (E)) presented with typical papillary architecture, isomorphous tumour cells, and nuclei; no mitoses are detectable; (f) low-grade oligodendroglioma (WHO grade II, sample (F)) with the characteristic honeycomb pattern (similar to Figure 1(b)); (g) features of anaplastic oligodendroglioma (WHO grade III, sample (G)) consists of a honeycomb pattern similar to low-grade oligodendroglioma, higher cellularity, and pleomorphic nuclei and occasional mitoses (arrow); (h) glioblastoma (WHO grade IV, sample (H)) as a highly cellular and polymorphous glial tumour with vascular proliferation (arrow) and multinucleated cells. Scale bar in (a) is valid for (a)-(h), $50 \mu \mathrm{m}$. 


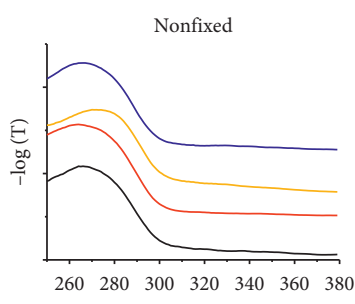

Wavelength (nm)

(a)

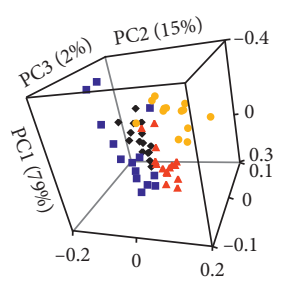

Sample A -

Sample B

Sample C A

Sample D •

(e)

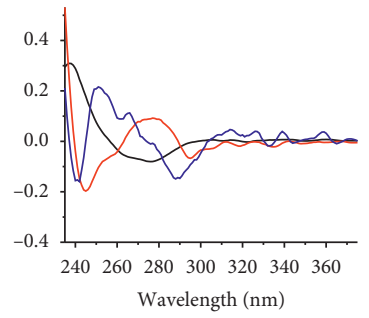

Wavelength (nm)

$-\quad \mathrm{PC}$

\begin{tabular}{rr}
- \\
\hline
\end{tabular}

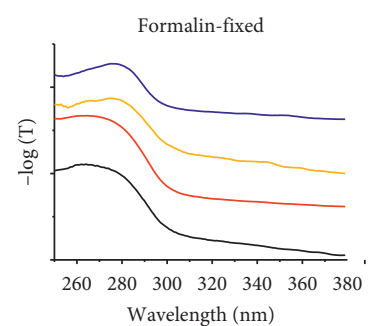

(b)

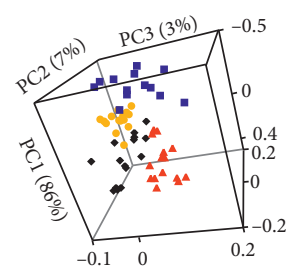

Sample A

Sample B •

Sample C 4

Sample D *

(f)

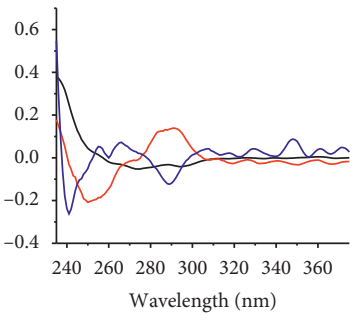

$-\mathrm{PCl}$

- PC2

(i)

(j)

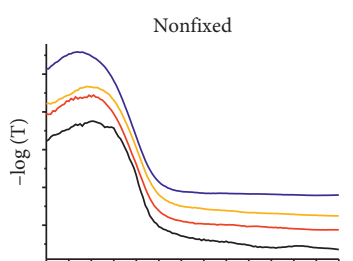

$\begin{array}{lllllll}260 & 280 & 300 & 320 & 340 & 360 & 380\end{array}$ Wavelength (nm)

(c)

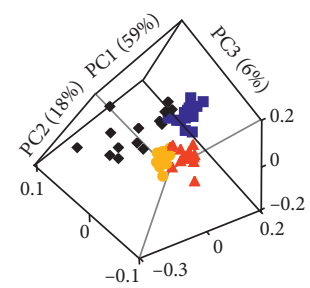

Sample E -

Sample F •

Sample G A

Sample H •

(g)

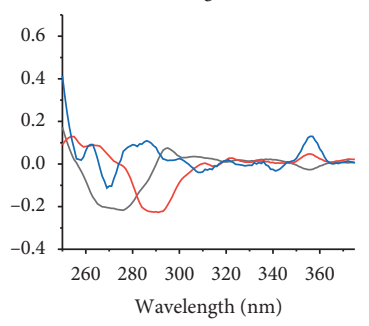

$\begin{array}{ll}- & \mathrm{PC} 1 \\ - & \mathrm{PC} 2 \\ - & \mathrm{PC} 3\end{array}$

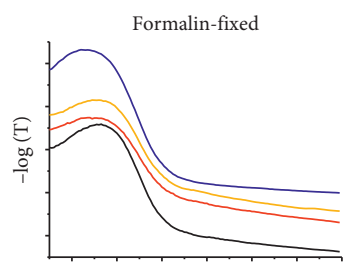

$\begin{array}{lllllll}260 & 280 & 300 & 320 & 340 & 360 & 380\end{array}$ Wavelength (nm)

(d)

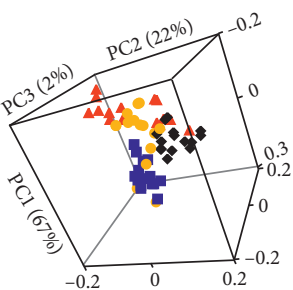

Sample E -

Sample F

Sample G A

Sample H •

(h)

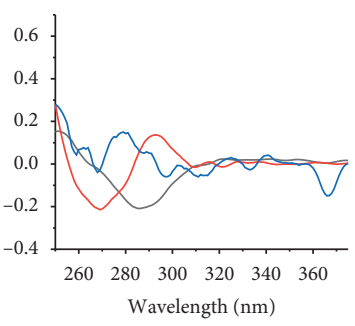

$\mathrm{PC} 1$
$-\quad \mathrm{PC} 2$

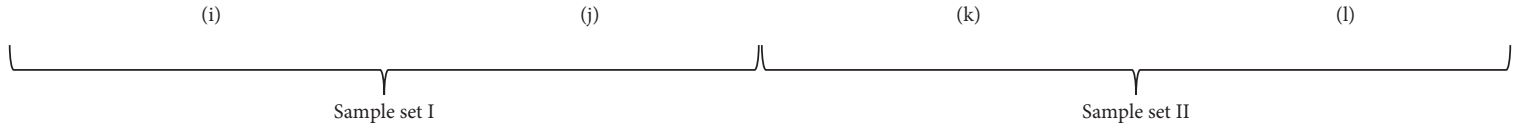

FIgURe 2: UV mean spectra and corresponding PCA models: UV absorption mean spectra of nonfixed (a, c) and formalin-fixed (b, d) brain tumour tissue cross sections in the range from $230 \mathrm{~nm}$ to $380 \mathrm{~nm}$. The spectra are vertically displaced, and SNV transformed. (e) and (g) 3D scores plot with PC1 versus PC2 versus PC3 to differentiate between nonfixed tissue samples. (f) and (h) 3D scores plot with PC1 versus PC2 versus PC3 to differentiate between formalin-fixed tissue samples. The representative tumour entities for the tissue samples A to D with different malignancies are fibrous meningioma (sample (A)), oligodendroglioma (sample (B)), anaplastic ependymoma (sample (C)), and glioblastoma (sample (D)). The representative tumour entities for the tissue samples $\mathrm{E}$ to $\mathrm{H}$ with different malignancies are plexus papilloma (sample (E)), oligodendroglioma (sample (F)), anaplastic oligodendroglioma (sample $(\mathrm{G})$ ), and glioblastoma (sample (H)). Corresponding loadings plots of PC1 (black), PC2 (red), and PC3 (blue) for nonfixed (i, k) and formalin-fixed (j, l) cross sections.

tumour cross sections. For the comparison of the quality of nonfixed and fixed models, each PCA is combined with a Bayesian discriminant analysis with Mahalanobis distance. The accordance of the model's prediction with the pathologist's assumption is $88 \%$ for the nonfixed sample set I and $72 \%$ for the nonfixed sample set II. 98\% accuracy was obtained for the formalin-fixed sample set I and $98 \%$ for the sample set II (Tables S9-S12).

3.4. Fourier-Transform Infrared Microspectroscopy of Formalin-Fixed and Nonfixed Human Brain Tumours. Figures 4(a)-4(d) show the FTIR mean spectra of the human brain tumours for each tissue sample. The wavenumbers between $2410 \mathrm{~cm}^{-1}$ and $2240 \mathrm{~cm}^{-1}\left(\mathrm{CO}_{2}\right.$ in air) are excluded. For each tissue cross section, there is a dominant band between $3670 \mathrm{~cm}^{-1}$ and $3200 \mathrm{~cm}^{-1}$ followed by a double band at $2930 \mathrm{~cm}^{-1}$ and $2850 \mathrm{~cm}^{-1}$ which is not pronounced for the fibrous meningioma, sample A. For the fingerprint region from $1780 \mathrm{~cm}^{-1}$ to $700 \mathrm{~cm}^{-1}$, the mean spectra show the same trend, except for the band at $1068 \mathrm{~cm}^{-1}$ which is dominant for samples BD. The spectra of nonfixed cross sections (Figures 4(a) and 4(c)) and formalin-fixed ones (Figures 4(b) and 4(d)) are mostly comparable.

Sample $G$ reveals small spectral differences between $3000 \mathrm{~cm}^{-1}$ to $3700 \mathrm{~cm}^{-1}$, whereas sample $\mathrm{H}$ displays spectral changes between $3500 \mathrm{~cm}^{-1}$ to $3100 \mathrm{~cm}^{-1}$ for nonfixed and fixed cross sections. In order to highlight these differences, derivative spectra were generated (Figure S4). 


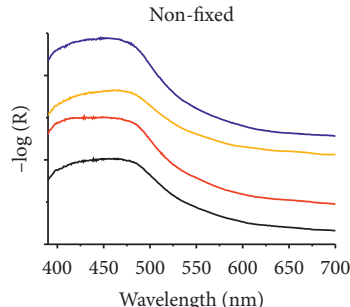

(a)

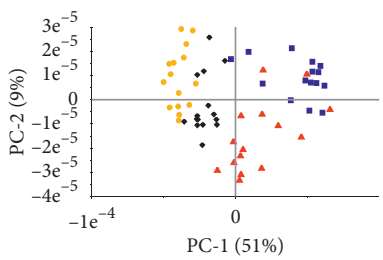

Sample A

Sample B

Sample C A

Sample D •

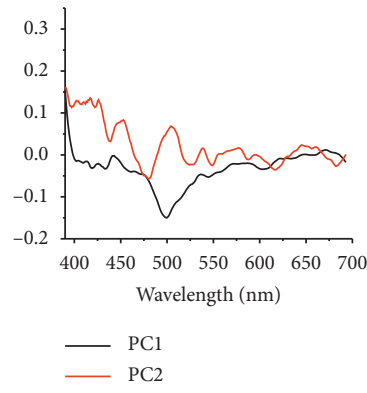

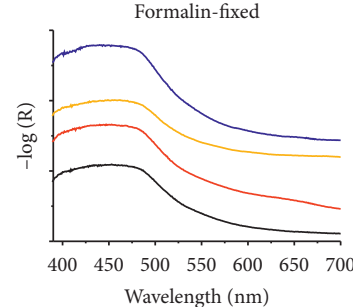

(b)

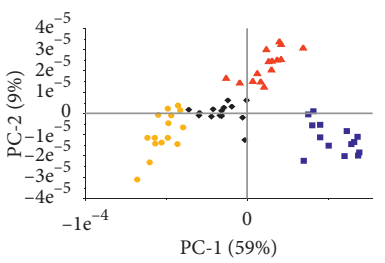

Sample A -

Sample B o

Sample C A

Sample D •

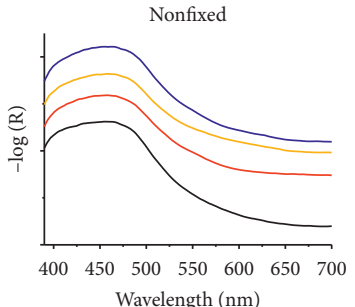

(c)

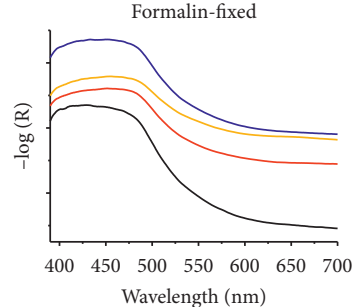

(d)

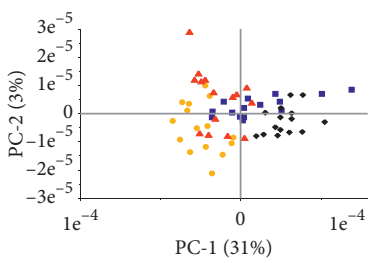

Sample E -

Sample F

Sample G ^

Sample H •

(g)

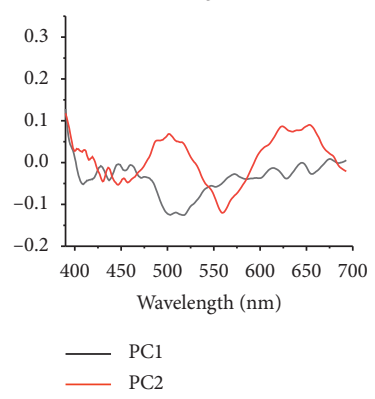

(k)

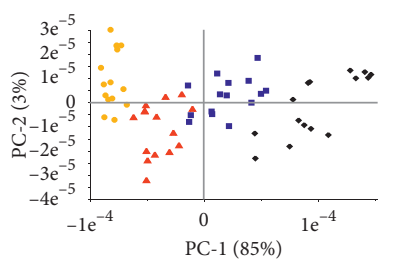

Sample E ㅁ

Sample F 。

Sample G A

Sample H •

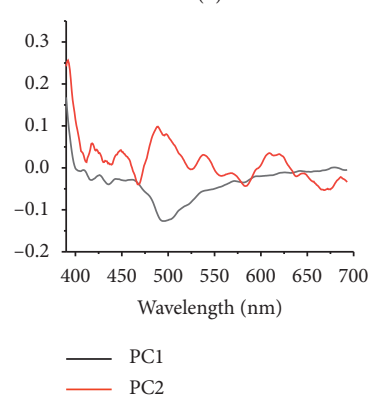

(l) (f)

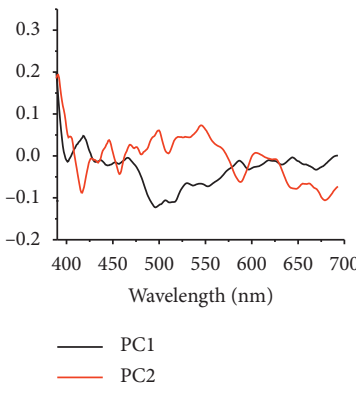

(j)

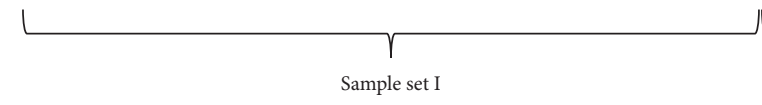

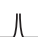

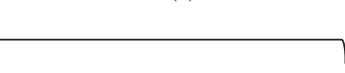

FIGURE 3: ELS mean spectra and corresponding PCA models: ELS mean spectra of nonfixed (a, c) and formalin-fixed (b, d) brain tumour tissue cross sections in the VIS from $380 \mathrm{~nm}$ to $700 \mathrm{~nm}$. The spectra are vertically displaced and area normalized. (e, g) Scores plot with PC1 versus PC2 to differentiate between nonfixed tissue samples. $(f, h)$ Scores plot with PC1 versus PC2 to differentiate between formalin-fixed tissue samples. The representative tumour entities for the tissue samples A to D with different malignancies are fibrous meningioma (sample (A)), oligodendroglioma (sample (B)), anaplastic ependymoma (sample (C)), and glioblastoma (sample (D)). The representative tumour entities for the tissue samples $\mathrm{E}$ to $\mathrm{H}$ with different malignancies are plexus papilloma (sample (E)), oligodendroglioma (sample (F)), anaplastic oligodendroglioma (sample (G)), and glioblastoma (sample (H)). Corresponding loadings plots of PC1 (black) and PC2 (red) for nonfixed (i, k) and formalin-fixed (j, l) cross sections.

Figures 4(e) to 4(l) show the PCA models of the preprocessed spectra and allows a differentiation of the tissues for nonfixed and formalin-fixed cross sections. The scores plot of the nonfixed brain tumour cross sections sample set I is shown in Figure 4(e). PC1 (57\% explained variance) separates sample A and sample B from sample $C$ and sample D. The tissue samples C and D are overlapping. PC2 (15\% explained variance) distinguishes the tissue samples $\mathrm{C}$ and $\mathrm{D}$ from each other. The scores plot of PC1 (71\% explained variance) versus PC2 (9\% explained variance) of the formalin-fixed brain tumour cross sections sample set $I$ is shown in Figure 4(f). Here, the tissue samples B and D are overlapping on $\mathrm{PC} 1$, while the tissue samples $\mathrm{A}$ and $\mathrm{C}$ are separated. PC2 ensures the separation of sample D from sample B. Overall, we find less overlap between the clusters in the scores plot of the formalin-fixed samples. Four measurements of sample D are located in the group of sample B. The corresponding loadings plots are shown in Figure 4(i) for the nonfixed model and in Figure 4(j) for the formalin-fixed model sample set I. The main variance of nonfixed and formalin-fixed cross sections is for PC1 between $3000 \mathrm{~cm}^{-1}$ and $2800 \mathrm{~cm}^{-1}$ and at $1635 \mathrm{~cm}^{-1}$. For PC2 of the formalin-fixed model, the region between $3000 \mathrm{~cm}^{-1}$ and $2800 \mathrm{~cm}^{-1}$ is again dominant as well as the bands at $1700 \mathrm{~cm}^{-1}, 1621 \mathrm{~cm}^{-1}$, and $1557 \mathrm{~cm}^{-1}$. Compared to PC2 of the nonfixed model, the main variance depends on the bands at $1700 \mathrm{~cm}^{-1}, 1621 \mathrm{~cm}^{-1}$, and $1557 \mathrm{~cm}^{-1}$. The loadings plots of PC1 have nearly the same shape and the main variances for both nonfixed and formalin-fixed cross sections. Comparable PCA models could be achieved with sample set II for 


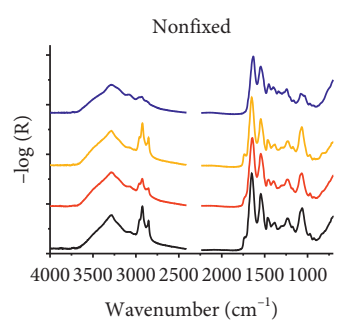

(a)

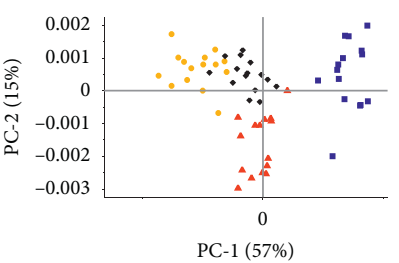

Sample A -

Sample B -

Sample C A

Sample D •

(e)
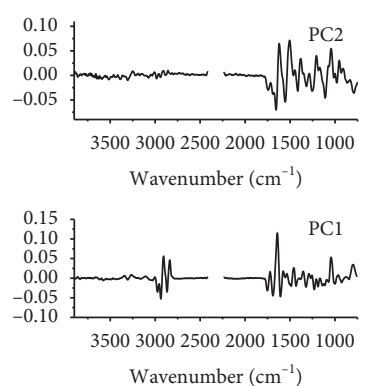

(i)

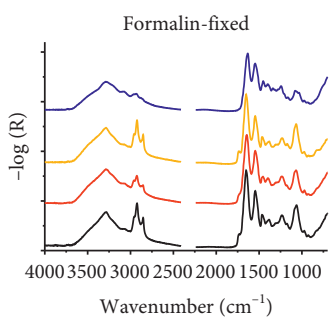

(b)

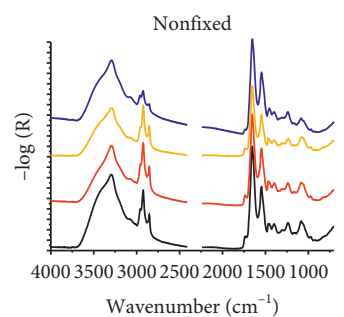

(c)

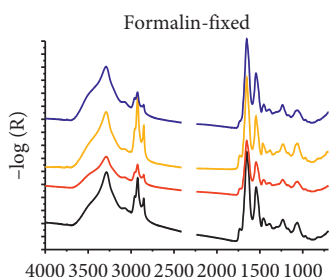

Wavenumber $\left(\mathrm{cm}^{-1}\right)$

(d)

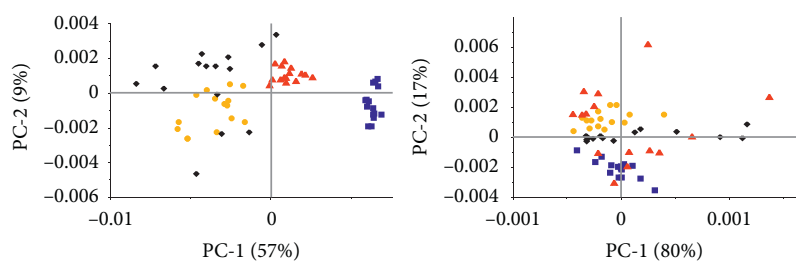

Sample E घ

Sample F -

Sample G $\Delta$

Sample H •

(g)

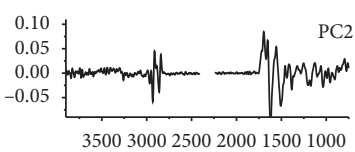

Wavenumber $\left(\mathrm{cm}^{-1}\right)$

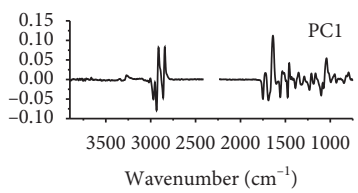

(j)

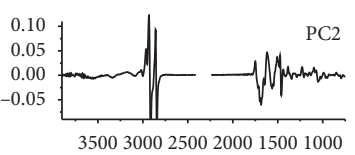

Wavenumber $\left(\mathrm{cm}^{-1}\right)$

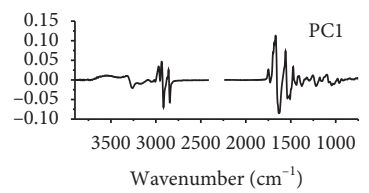

(k)

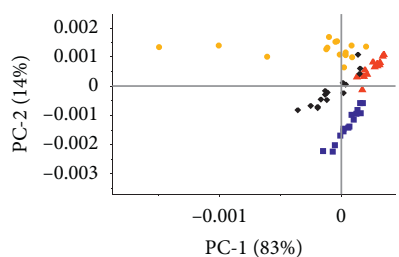

Sample E

Sample F

Sample G

Sample H

(h)

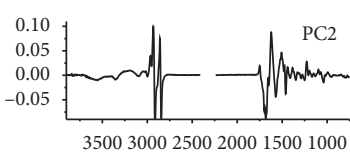

Wavenumber $\left(\mathrm{cm}^{-1}\right)$

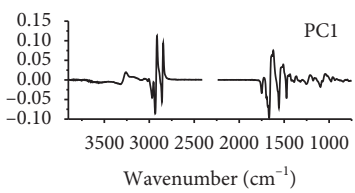

Sample set I

Sample set II

FIGURE 4: FTIR mean spectra and corresponding PCA models: FTIR mean spectra of nonfixed (a, c) and formalin-fixed (b, d) brain tumour tissue cross sections from $4000 \mathrm{~cm}^{-1}$ to $700 \mathrm{~cm}^{-1}$. The wavenumbers of the $\mathrm{CO}_{2}$ band between $2410 \mathrm{~cm}^{-1}$ and $2240 \mathrm{~cm}^{-1}$ were excluded. The spectra are vertically displaced and unit vector normalized. (e, g) Scores plot with PC1 versus PC2 to differentiate between nonfixed tissue samples. (f, h) Scores plot with PC1 versus PC2 to differentiate between formalin-fixed tissue samples. The representative tumour entities for the tissue samples A to D with different malignancies are fibrous meningioma (sample (A)), oligodendroglioma (sample (B)), anaplastic ependymoma (sample (C)), and glioblastoma (sample (D)). The representative tumour entities for the tissue samples $\mathrm{E}$ to $\mathrm{H}$ with different malignancies are plexus papilloma (sample (E)), oligodendroglioma (sample (F)), anaplastic oligodendroglioma (sample (G)), and glioblastoma (sample (H)). Corresponding loadings plots of PC1 (black) and PC2 (red) for nonfixed (i, k) and formalin-fixed (j, l) cross sections.

the nonfixed (Figures $4(\mathrm{~g})$ and $4(\mathrm{k}))$ and the formalin-fixed (Figures 4(h) and 4(l)) brain tumour cross sections.

For the quality comparison of nonfixed and fixed models, each PCA was combined with a Bayesian discriminant analysis with Euclidian distance. In accordance with the model's prediction with the pathologist's assumption, the accuracy is $93 \%$ for the nonfixed sample set I and $87 \%$ for nonfixed sample set II. An accuracy of $97 \%$ was achieved for the formalin-fixed sample set I and sample set II reached an overall accuracy of 93\% (Tables S13-S16).

3.5. Combining Datasets from Nonfixed and Formalin-Fixed Samples. For each spectroscopic method, we further combined the spectra of the nonfixed and formalin-fixed cross sections in one dataset. Based on this dataset combination, a multivariate model was built to analyse the influence of the fixation step on the clustering. This could result in the following options: we get two main clusters caused by the dominant information of the fixation (nonfixed and formalin-fixed), where the tissue samples are not located on the first PC axis of maximum variance. The second option is that the tissue sample information is located on the first PC, resulting in four main clusters (samples A, B, C, and $D$ and samples E, F, G, and H). In the case of the second option, it can be excluded that the consecutive brain tumour cross sections are too inhomogeneous.

The results of this dataset combination are illustrated in the PCA scores and loadings plots shown in the supplementary material (available here) (Figures S5-S7). Regardless of the fixation, each spectroscopic method results in a clustering according to the different tissue samples. 
For the PCA model of the UV spectra, the clustering and assignment of the tissue samples of both data sets are possible by building the model with four PCs. The explained variances of PC1 to PC4 describe $97 \%$ for sample set I and $92 \%$ for sample set II of the data. The overall accuracy is $91 \%$ (I) and $84 \%$ (II). For the PCA model of the ELS spectra, the clustering and assignment of the tissues are possible by building the model with three PCs. The sum of the explained variance of PC1 to PC3 is $67 \%$ for sample set I. For sample set II, we need four PCs and the explained variance is $79 \%$. The overall accuracy is $82 \%$ for sample set I and $87 \%$ for sample set II. For the PCA model of the FTIR spectra, the clustering and assignment of the tissue samples are possible by building the model with four PCs. The sum of the explained variance of PC1 to PC4 is $86 \%$ for sample set I and $98 \%$ for sample set II. The overall accuracy is $92 \%$ (I) and $87 \%$ (II). Overall, models built of all three spectroscopic methods for nonfixed and formalin-fixed cross sections allow a characterization according to the tissue samples. We ascertained that the results for the formalin fixation are, independently of the spectroscopic techniques, very close to the ones for nonfixed samples. Otherwise, a combined model building would not be possible in this manner.

\section{Discussion}

We investigated a multimodal combination of UV, ELS, and FTIR microspectroscopy to obtain laterally resolved information of primary brain tumour tissues. Formalin fixation yields even better diagnostic results than native samples for the generation of spectroscopic multimodal datasets. Using this multimodal approach, UV and FTIR spectroscopy acquire the chemical information of the cross sections, whereas the ELS is linked to tissue morphology [33].

From a practical point of view, fixed and thus stable specimens are preferred for spectroscopy [54] and can even be used in retrospective analyses. Native brain tissue samples suffer from fast degradation processes but could be used in real-time methods. Usually, resected tissues are directly transferred to a buffered formalin solution in the clinical routine [9]. Our results demonstrate that nonfixed and formalin-fixed cross sections should deliver comparable results for brain tumour typing. This is important for a future transfer of spectroscopic results to intraoperative applications. For a verification of this requirement, a PCADA is used. The PCA structures data based on objective mathematical criteria and offers an unbiased view on the spectra $[44,51]$. This reduces the required data space and extracts the relevant optical information (biomarkers) for tumour characterization $[38,42]$.

4.1. Ultraviolet Absorption Microspectroscopy. UV spectroscopy measures electronic transitions from the ground state to the excited state of $\pi$-electrons or nonbonding electrons. UV light excites the electrons to higher antibonding molecular orbitals. The resulting spectra have broad bands. In combination with PCA and an appropriate spectral preprocessing, small chemical and morphological differences can be recognized on the brain tumour cross sections and used as biomarkers [5, 38]. The extraction of spectral features by appropriate data pretreatment is clearly visible in Figures 2(a)-2(d) (standard normal variate, SNV) and Figure S1 (SNV plus derivation). The authors consider UV spectroscopy as promising tool for further studies because it is inexpensive, easy to utilize, and offers chemical information based on the sum of biological molecules [38]. In the UV spectra, broad absorption bands between $230 \mathrm{~nm}$ and $300 \mathrm{~nm}$ are visible and mainly assigned to changes in the DNA and protein absorption. Additionally, the contained aromatic amino acids have distinct absorption bands in the UV (phenylalanine at $257 \mathrm{~nm}$, tyrosine at $274 \mathrm{~nm}$, and tryptophan at $280 \mathrm{~nm}$ ) [63].

Due to protein cross-linking caused by fixation, spectral differences could appear in fixed vs. nonfixed UV spectra. This might be visible for sample $B$ and sample $H$.

The corresponding loadings of PC1 (Figures 2(i)-2(l)) can be described by a protein component at around $240 \mathrm{~nm}$ [38]. Another protein component absorbs at $280 \mathrm{~nm}$, whereas the DNA component (including histones) absorbs at $260 \mathrm{~nm}[38,63]$. We assume that the wavelength shift of the formalin-fixed model by $5 \mathrm{~nm}$ to longer wavelengths is related to PC2 and could be due to environmental changes. The cross-linking is based on a reaction of the formalin with free amino groups like lysine, cysteine, histidine, arginine, and tyrosine and the reactive hydroxyl groups of serine and threonine in proteins and nucleotides. It further interacts with $\mathrm{C}=\mathrm{C}$ and $-\mathrm{SH}$ in unsaturated lipids $[1,5]$. Human brain tissue has high lipid content [7]. The tissue degradation process can be accelerated by the high energy of UV excitation light. Obviously, the fixation inhibits the degeneration of tissue resulting in a less heterogeneous clustering.

4.2. Elastic Light Scattering Microspectroscopy. The ELS mainly characterizes sample texture and morphology. For structures such as cells and subcellular organelles, it is based on the magnitude of Mie scattering, where the cells and organelles are acting as microscopic and sub-microscopic optical resonators $[33,64]$. Chromosomes as part of the nucleus can be regarded as structured particle arrays [65]. Arrangements of many cells are forming tissues. The tissues contain the scattering centres of the subunits. The resulting spectra of the tissues are closely linked to their morphology $[33,34,64]$ : this ELS information is complementary to the chemical information of UV and FTIR microspectroscopy. Additionally, ELS is one of the most inexpensive methods.

Formalin fixation preserves morphological structures; therefore, spectral differences are not likely to emerge. The differences in sample $\mathrm{C}$ could result from degeneration of the nonfixed tissue.

As mentioned above, spectral differences between tissue types are often small. Marginal variations in the particle size and shape or the refractive indices result in complex spectral signatures and are nonrandom [33, 65]. Therefore, data preprocessing and multivariate data analysis are needed to enhance superimposed small information in the spectra $[44,51,65]$. Figure S3 shows the ELS mean spectra after area 
normalization and first derivation as a part of the spectral preprocessing before calculating a PCA. As a result, the tiny differences in the microstructures and sub-microstructures lead to different spectral signatures and hence the underlying modulation can be detected. The corresponding loadings of the PCs are carrying the information correlated to the histopathology. The first PCs in Figures 3(i)-3(l) show a curve with one broad global peak having a negative maximum at $500 \mathrm{~nm}$. This can be assigned to the scattered light [66]. The overall signature of the curve resembles the first derivative (Figure S3). Additionally, a ripple structure with less intensity is overlaid. This ripple structure is a repeated pattern and represents the main variance of the loadings of PC2. It can be assigned to Mie interference resulting from the texture of the cross sections [33]. The fixation procedure leads to a cross-linking of the proteins, which manifests itself in small texture changes. This is evident in the shape of the loadings of PC2. The main variance, the separation of the tissue samples, is given on PC1, which looks similar in the nonfixed and formalin-fixed models. Even the order of the samples in the scores plots on PC1 is identical for sample set I and comparable for sample set II (Figures 3(e)-3(h)). The main aim of fixation is to maintain intact morphological features $[12,15,18]$; hence, we have fewer divergences in the ELS models in the main variance. However, on PC2, we see a few differences in the loadings as well as in the scores plots. This might be caused by minor changes in the texture through cross-linking of proteins. The ELS is known for its particular high sensitivity [66]. For example, the earliest possible detection of carcinogenesis in tissues is feasible by ELS $[35,36]$, as shown in a rat model six weeks prior to early detectable biomarkers [37]. The variance within the measured cross sections is higher for the native tissue than for formalin-fixed tissue.

4.3. Fourier-Transform Infrared Microspectroscopy. IR spectroscopy is a common method to measure the chemical information by acquiring molecular vibrations. A labelling of samples is not necessary, and their preparation is minimal. Hence, we choose FTIR microspectroscopy as the third method, because it is well described as a standard method for tissue characterization [7]. The extraction of spectral features by appropriate data pretreatment is clearly visible in Figures 4(a)-4(d) (vector normalization) and Figure S4 (vector normalization plus derivation).

Formalin fixation of tissue results in structural alterations in macromolecules present in the tissues. The most prominent change induced by this process is protein crosslinking, leading to an overemphasis of protein-related bands such as amide I and amide II vibrations. The practical benefit of the structural stabilization of the tissue by protein crosslinking is a more robust PCA model for the characterization of the tumour entities. Thus, the spectral differences of sample $G$ between fixed and nonfixed IR spectra can be deduced from an alteration in protein and additionally in lipid content. For sample $\mathrm{H}$, the $\mathrm{OH}$-band is more pronounced for the nonfixed sample compared to the fixed one since the fixation process causes tissue dehydration.
The spectral band assignment for brain tumours is summarized in Table 1. In the wavenumber range between $2850-2960 \mathrm{~cm}^{-1}$, a strong influence is recognizable which is attributed to fatty acids ( $v(\mathrm{CH}), v(\mathrm{CH} 2), v(\mathrm{CH} 3))[32,67]$. Additionally, $\nu(\mathrm{C}=\mathrm{O})$ at $1750 \mathrm{~cm}^{-1}$ is connected to lipids $[32,68,69]$. The human brain tissue consists of fatty acids and lipids [7], and the formation of gliomas is linked to significant changes in fat metabolism [32]. Therefore, the variation of lipid concentration and composition during tumour genesis also affects the spectroscopic properties tumour. The prominent bands around the amide $\mathrm{I}$ and amide II vibrations $\left(1655 \mathrm{~cm}^{-1}, 1582 \mathrm{~cm}^{-1}\right.$, and $\left.1547 \mathrm{~cm}^{-1}\right)$ are assigned to proteins and peptides [32, 67-71]. An example is collagen fibers for the use of brain tumour detection with IR spectroscopy [70]. In most cases of tumour genesis, genetic changes are present. The activation of oncogenes and the deactivation of tumour suppressors are essential. Further, the methylation of DNA is not negligible [72]. DNA and RNA changes are associated in IR microspectroscopy with phosphate and/or phosphate diester at $1234 \mathrm{~cm}^{-1}$ and $1063 \mathrm{~cm}^{-1}\left(\nu\left(\mathrm{PO}_{2}\right)\right)$, with $1165 \mathrm{~cm}^{-1}(\nu$ $(\mathrm{C}-\mathrm{O}))$, and with $1040 \mathrm{~cm}^{-1}$ to $1110 \mathrm{~cm}^{-1}$ ( $\nu$ (C-O)) $[32,67,68,71]$. The range between $1040 \mathrm{~cm}^{-1}$ to $1110 \mathrm{~cm}^{-1}$ is most likely associated to $v(\mathrm{C}-\mathrm{O})$ stretching vibrations from ribose $v(\mathrm{C}-\mathrm{O})$ skeletal vibrations in RNA and DNA. Additionally, the symmetric stretching vibration of the nucleic acids phosphodiester group strongly correlates to changes in DNA and RNA [71].

4.4. Combining Datasets from Nonfixed and Formalin-Fixed Samples. Additionally, we build multivariate models for each spectroscopic method combining the formalin-fixed and nonfixed tissue datasets. Since adjacent tissue cross sections were either formalin-fixed or remained native, the nonfixed cross sections are not exactly identical to the formalin-fixed ones. Consequently, the pathologist needed to examine each cross section individually to ensure a visual matching of nonfixed and fixed tissue samples. Furthermore, brain tumours are inhomogeneous tissues, resulting in even greater structural differences between consecutive cross sections.

The distinction of the samples according to their malignancy is demonstrated not only with the singular models of each preparation technique but also with the combined model containing nonfixed and formalin-fixed spectra. The dominating effect of the clustering in the combined models is not the preparation procedure (nonfixed and formalinfixed) but the different tissue samples with varying malignancies. Based on this observation, we assume that the nonfixed and formalin-fixed sections of each brain tumour are comparable.

In Table 2, all calculated model quality parameters for each model are summarized. All values are calculated from the confusion matrix. The accuracy marks the accordance of the model's prediction with the pathologist's assignment. As all classes have the same size, they are treated equally. Therefore, the sensitivity, specificity, false positive rate, and precision are average values for each 
TABLE 1: Spectral band assignments for brain tumors for the FTIR models.

\begin{tabular}{|c|c|c|c|}
\hline Band position $\left(\mathrm{cm}^{-1}\right)$ & Assignments & Remarks & References \\
\hline $2960-2850$ & $v(\mathrm{CH}), v\left(\mathrm{CH}_{2}\right), v\left(\mathrm{CH}_{3}\right)$ & Fatty acids & {$[32,67]$} \\
\hline$\sim 1750$ & $\mathrm{~N}(\mathrm{C}=\mathrm{O})$ & Lipids & {$[32,68,69]$} \\
\hline$\sim 1655, \sim 1582, \sim 1547$ & $v(\mathrm{C}=\mathrm{O}), v(\mathrm{C}-\mathrm{N})$ & Amide I and amide II of proteins and peptides & {$[32,67-71]$} \\
\hline$\sim 1415$ & $v(-\mathrm{COO})$ & Amino acid side chain & [71] \\
\hline $1455-1460$ & $v\left(\mathrm{CH}_{3}\right)$ & Amino acid side chain & {$[32,67,71]$} \\
\hline$\sim 1465, \sim 1385$ & $v\left(\mathrm{CH}_{2}\right), v\left(\mathrm{CH}_{3}\right)$ & Cholesterol, phospholipids & {$[32]$} \\
\hline 1234,1063 & $v\left(\mathrm{PO}_{2}\right)$ & Phosphate, phosphodiesters of RNA and DNA & {$[32,67,68,71]$} \\
\hline$\sim 1165$ & $v(\mathrm{C}-\mathrm{O})$ & RNA and/or carbohydrates & [71] \\
\hline$\sim 1200, \sim 1170$ & $v(\mathrm{C}-\mathrm{O})$ & Proteins & {$[32]$} \\
\hline$\sim 12$ & $v(\mathrm{C}-\mathrm{O}), v(\mathrm{C}-\mathrm{C}), v(\mathrm{C}-\mathrm{O}-\mathrm{H}), v(\mathrm{C}-\mathrm{O}-\mathrm{C})$ & Carbc & {$[67,68]$} \\
\hline $1040-1110$ & $v(\mathrm{C}-\mathrm{O})$ & RNA and DNA & [71] \\
\hline
\end{tabular}

TABLE 2: Overview of model quality parameters: ccuracy, sensitivity, specificity, false positive rate, and precision are calculated for each method and model.

\begin{tabular}{|c|c|c|c|c|c|c|c|}
\hline & & & Accuracy $/ \%$ & Sensitivity/\% & Specificity/\% & False positive rate $/ \%$ & Precision/\% \\
\hline \multirow{9}{*}{ Sample set I } & \multirow{3}{*}{ UV absorption } & Nonfixed & 82 & 82 & 94 & 6 & 89 \\
\hline & & Fixed & 95 & 95 & 98 & 2 & 96 \\
\hline & & Combined & 91 & 91 & 97 & 3 & 91 \\
\hline & \multirow{3}{*}{ Elastic light scattering } & Nonfixed & 88 & 88 & 96 & 4 & 88 \\
\hline & & Fixed & 98 & 98 & 99 & 1 & 98 \\
\hline & & Combined & 92 & 92 & 97 & 3 & 92 \\
\hline & \multirow{3}{*}{ FTIR absorption } & Nonfixed & 93 & 93 & 98 & 2 & 94 \\
\hline & & Fixed & 97 & 97 & 99 & 1 & 97 \\
\hline & & Combined & 92 & 92 & 97 & 3 & 92 \\
\hline \multirow{9}{*}{ Sampel set II } & \multirow{3}{*}{ UV absorption } & Nonfixed & 85 & 85 & 95 & 5 & 88 \\
\hline & & Fixed & 75 & 75 & 92 & 8 & 79 \\
\hline & & Combined & 84 & 84 & 94 & 5 & 84 \\
\hline & \multirow{3}{*}{ Elastic light scattering } & Nonfixed & 72 & 72 & 91 & 9 & 74 \\
\hline & & Fixed & 98 & 98 & 99 & 1 & 98 \\
\hline & & Combined & 79 & 79 & 93 & 7 & 82 \\
\hline & \multirow{3}{*}{ FTIR absorption } & Nonfixed & 87 & 87 & 96 & 4 & 87 \\
\hline & & Fixed & 93 & 93 & 98 & 2 & 94 \\
\hline & & Combined & 87 & 87 & 96 & 4 & 90 \\
\hline
\end{tabular}

In general, the models of formalin-fixed cross sections are better than the nonfixed models. But, in combination, they are still working in one model independent from the preparation procedure.

tissue sample in this model and can be calculated on the basis of the confusion matrices as shown in the supplementary materials (available here). The sensitivity or true positive rate means, when it is actually, through the pathologist's assumption, "yes," how often does the model predict it as "yes." The specificity describes, when it is actually "no," how often does the model predict it as "no." When the model predicts a tissue sample as "yes," how often this prediction is correct is described by the precision. High values for sensitivity, specificity, and precision show a good model quality. On the other hand, a smaller false positive rate indicates a better model. According to Table 2, all models and methods exhibit values better than $70 \%$ for accuracy, sensitivity, and specificity. In statistics, these are acceptable values for biological samples [34]. In all cases, the formalin-fixed models are significantly more accurate and have a less variance as the nonfixed models. The authors identify the fast degradation of the nonfixed samples during the measuring process as a possible reason.
Comparing the spectroscopic methods vice versa, the FTIR spectroscopy yields the best quality parameters in sum, directly followed by the ELS spectroscopy. For the ELS spectroscopy, the model of the nonfixed cross sections has nearly $10 \%$ less good values than the formalin-fixed model. This is probably due to the sensitivity of the method, because it detects small alterations before they are visible with the eye [35-37, 66]. Overall, ELS more accurately detects the aging and degradation of the nonfixed cross sections than the other investigated techniques. The nonfixed model of the UV absorption spectra is possibly influenced by the same effect. The high energy of excitation light can accelerate the tissue's degradation process more than other excitation sources like a globar for the infrared. This assumption seems reasonable as we have a closely better UV spectroscopic-based model for the formalin-fixed cross sections.

Overall, most of the model quality parameters are slightly better for the formalin-fixed cross sections considering all three spectroscopic methods. This can also be observed for the PCA scores plots (Figures 2(e)-2(h), 3(e)- 
3(h), and 4(e)-4(h)) in terms of improved cluster separation and a less pronounced dispersion within each cluster.

\section{Conclusions}

We provide a proof of concept investigation of brain tumour tissues by a multimodal spectroscopy approach. Due to a combination of three different spectroscopic methods, an overall spectroscopic-based PCA-DA model was developed to characterize brain tumour tissues with regard to the degree of malignancy. Using this spectroscopic combinatorial approach, we demonstrated that formalin fixation is a suitable sample preparation method for spectroscopic investigations. Formalin fixation does not negatively affect the model clustering regarding the four tumour gradings and results in even improved PCA-DA models compared to the nonfixed dataset. Future research work will not only include the investigation of other tumour types but also consider the common paraffin embedding procedure. Therefore, we will compare paraffin-embedded formalin-fixed cross sections with native and formalin cross sections.

\section{Data Availability}

All raw data used in this manuscript are available on request from the corresponding author.

\section{Disclosure}

J.W. Bartsch, E. Ostertag, and R. Ritz have patents pending (WO002015097089A1 and EP000002887050A1).

\section{Conflicts of Interest}

The authors declare no conflicts of interest.

\section{Authors' Contributions}

MS, AL, JWB, $\mathrm{MBr}, \mathrm{BB}, \mathrm{SH}, \mathrm{RR}, \mathrm{KR}$, and $\mathrm{EO}$ were responsible for the conceptualization. MS, AL, JWB, AP, JS, $\mathrm{BB}, \mathrm{SH}, \mathrm{SN}, \mathrm{RR}$, and $\mathrm{EO}$ curated the data. $\mathrm{MS}, \mathrm{AL}, \mathrm{BB}, \mathrm{SH}$, and EO conducted the formal analysis:. MS, JWB, AP, JS, $\mathrm{SH}$, and $\mathrm{SN}$ investigated the study. MS, $\mathrm{AL}, \mathrm{BB}, \mathrm{SH}$, and $\mathrm{EO}$ were involved in the methodology. KR and EO were responsible for the project administration. JWB, RR, CN, MT, and $\mathrm{KR}$ collected the resources. JWB, MBr, and $\mathrm{EO}$ supervised the study. MS, AL, JWB, and EO validated the study. MS, AW, and EO were involved in the visualization. MS, AL, JWB, MBa, AW, MBr, and EO were involved in the writing of the original draft. MS, AL, JWB, MBa, AW, MBr, AP, JS, BB, SN, CN, MT, RR, KR, EO wrote, reviewed, and edited the manuscript.

\section{Acknowledgments}

The authors especially thank Yeliz Donat and Sabine Motzny for preparing the cross sections, Lieselotte Barac for data acquisition, and Prof. Waltraud Kessler and Prof. Dr. Rudolf Kessler for valuable discussions. Research reported in this publication was supported by the Baden-Württemberg
Ministry of Science, Research and Culture Ministerium (MWK), within the doctoral program of the University Tübingen "Intelligente Prozess-und Materialentwicklung in der Biomateriomics (IPMB)" (PhD student fellowships to MS and $\mathrm{MBa}$ ), and by the Baden-Wuerttemberg Stiftung. The article processing charge was funded by the BadenWürttemberg Ministry of Science, Research and Culture in the Funding Programme Open Access Publishing.

\section{Supplementary Materials}

In the manuscript, references to supplementary materials are given. Supplementary figures and tables are indicated by a leading "S" in their names. The supplementary material consists of an overview of the measured samples, the explanation of the confusion matrix terminology with examples, the derived mean spectra for each spectroscopic method, and the confusion matrices for each multivariate model. Further, the 2D-scores of the UV-models are included, and all multivariate models for a combined evaluation are shown in detail. (Supplementary Materials)

\section{References}

[1] G. Haroske, R. Zwönitzer, R. Zwönitzer, and P. Hufnagl, "Leitfaden digitale pathologie in der diagnostik," Der Pathologe, vol. 39, no. 3, pp. 216-221, 2018.

[2] T. Kalinski, H. Hofmann, R. Zwönitzer, J. Bernarding, and A. Roessner, "Virtuelle Mikroskopie und digitale Pathologie," Der Pathologe, vol. 27, no. 3, pp. 222-227, 2006.

[3] S. Al-Janabi, A. Huisman, and P. J. Van Diest, "Digital pathology: current status and future perspectives," Histopathology, vol. 61, no. 1, pp. 1-9, 2012.

[4] N. Lykkegaard Andersen, A. Brügmann, G. Lelkaitis, S. Nielsen, M. Friis Lippert, and M. Vyberg, "Virtual double staining: a digital approach to immunohistochemical quantification of estrogen receptor protein in breast carcinoma specimens," Applied Immunohistochemistry \& Molecular Morphology, vol. 26, no. 9, pp. 620-626, 2018.

[5] R. W. Kessler, Process Analytics: Strategies and Case Studies from Industrial Practice, John Wiley \& Sons, Hoboken, NJ, USA, 2012.

[6] I. W. Schie, J. Rüger, A. S. Mondol et al., "High-throughput screening Raman spectroscopy platform for label-free cellomics," Analytical Chemistry, vol. 90, no. 3, pp. 2023-2030, 2018.

[7] R. Salzer and H. W. Siesler, Infrared and Raman Spectroscopic Imaging, John Wiley \& Sons, Hoboken, NJ, USA, 2014.

[8] H. Krüger-Brand, "Digitization in the hospital: the infrastructure lacks funding," Deutsches Ärzteblatt, vol. 114, p. 48, 2017.

[9] D. Berg, K. Malinowsky, B. Reischauer, C. Wolff, and K. F. Becker, "Use of formalin-fixed and paraffin-embedded tissues for diagnosis and therapy in routine clinical settings," Methods in Molecular Biology, vol. 85, pp. 109-122, 2011.

[10] W. E. Grizzle, J. L. Fredenburgh, and R. B. Myers, "Fixation of tissues," Theory and Practice of Histological Techniques, vol. 6, pp. 53-74, 2008.

[11] R. Thavarajah, V. Mudimbaimannar, U. Rao, K. Ranganathan, and J. Elizabeth, "Chemical and physical basics of routine formaldehyde fixation," Journal of Oral and Maxillofacial Pathology, vol. 16, no. 3, p. 400, 2012. 
[12] J. A. Kiernan, "Formaldehyde, formalin, paraformaldehyde and glutaraldehyde: what they are and what they do," Microscopy Today, vol. 8, no. 1, pp. 8-13, 2000.

[13] S. M. Hewitt, F. A. Lewis, Y. Cao et al., "Tissue handling and specimen preparation in surgical pathology: issues concerning the recovery of nucleic acids from formalin-fixed, paraffinembedded tissue," Archives of Pathology \& Laboratory Medicine, vol. 132, no. 12, pp. 1929-1935, 2008.

[14] C. H. Fox, F. B. Johnson, J. Whiting, and P. P. Roller, "Formaldehyde fixation," Journal of Histochemistry \& Cytochemistry, vol. 33, no. 8, pp. 845-853, 1985.

[15] W. E. Grizzle, "Special symposium: fixation and tissue processing models," Biotechnic \& Histochemistry: official publication of the Biological Stain Commission, vol. 84, no. 5, pp. 185-193, 2009.

[16] W. E. Grizzle, "The use of fixatives in diagnostic pathology," Journal of Histotechnology, vol. 24, no. 3, pp. 151-152, 2001.

[17] I. Eltoum, J. Fredenburgh, and W. E. Grizzle, "Advanced concepts in fixation: 1 . effects of fixation on immunohistochemistry, reversibility of fixation and recovery of proteins, nucleic acids, and other molecules from fixed and processed tissues. 2. developmental methods of fixation," Journal of Histotechnology, vol. 24, no. 3, pp. 201-210, 2001.

[18] I. Eltoum, J. Fredenburgh, R. B. Myers, and W. E. Grizzle, "Introduction to the theory and practice of fixation of tissues," Journal of Histotechnology, vol. 24, no. 3, pp. 173-190, 2001.

[19] D. N. Louis, H. Ohgaki, O. D. Wiestler et al., "The 2007 WHO classification of tumours of the central nervous system," Acta Neuropathologica, vol. 114, no. 2, pp. 97-109, 2007.

[20] D. N. Louis, A. Perry, G. Reifenberger et al., "The 2016 world health organization classification of tumors of the central nervous system: a summary," Acta Neuropathologica, vol. 131, no. 6, pp. 803-820, 2016.

[21] H. Ohgaki, "Epidemiology of brain tumors," Methods in Molecular Biology, vol. 472, pp. 323-342, 2009.

[22] O. L. Chinot, W. Wick, W. Mason et al., "Bevacizumab plus radiotherapy-temozolomide for newly diagnosed glioblastoma," New England Journal of Medicine, vol. 370, no. 8, pp. 709-722, 2014.

[23] T. Engelhorn, N. E. Savaskan, M. A. Schwarz et al., "Cellular characterization of the peritumoral edema zone in malignant brain tumors," Cancer Science, vol. 100, no. 10, pp. 1856-1862, 2009.

[24] C. Nimsky, O. Ganslandt, P. Hastreiter, and R. Fahlbusch, "Intraoperative compensation for brain shift," Surgical Neurology, vol. 56, no. 6, pp. 357-364, 2001.

[25] I. Yang, M. Udawatta, G. N. Prashant et al., "Stereotactic radiosurgery for neurosurgical patients: a historical review and current perspectives," World Neurosurgery, vol. 122, pp. 522-531, 2019.

[26] R. Ritz, H. T Wein, K Dietz et al., "Photodynamic therapy of malignant glioma with hypericin: comprehensive in vitro study in human glioblastoma cell lines," International Journal of Oncology, vol. 30, no. 3, pp. 659-667, 2007.

[27] R. W. Kessler, "Spectral imaging: perspectives for fast, labelfree characterization of biological materials," BioPhotonik, vol. 2, pp. 30-34, 2010.

[28] F. Braun, Cancer detection by multispectral tissue examination, Ph.D thesis, Medical Faculty Mannheim University, Mannheim, Germany, 2019.

[29] É. Lemoine, F. Dallaire, R. Yadav et al., "Feature engineering applied to intraoperativein vivoRaman spectroscopy sheds light on molecular processes in brain cancer: a retrospective study of 65 patients," The Analyst, vol. 144, no. 22, pp. 6517-6532, 2019.

[30] C. Krafft, N. Bergner, B. Romeike et al., "Raman spectroscopic imaging as complementary tool for histopathologic assessment of brain tumors," Photonic Therapeutics and Diagnostics VIII, vol. 8207, 2012.

[31] T. Meyer, N. Bergner, B. Romeike et al., "Nonlinear microscopy, infrared, and Raman microspectroscopy for brain tumor analysis," Journal of Biomedical Optics, vol. 16, no. 2, Article ID 021113, 2011.

[32] N. Amharref, A. Beljebbar, S. Dukic et al., "Brain tissue characterisation by infrared imaging in a rat glioma model," Biochimica et Biophysica Acta (BBA)-Biomembranes, vol. 1758, no. 7, pp. 892-899, 2006.

[33] E. Ostertag, M. Stefanakis, K. Rebner, and R. W. Kessler, "Elastic and inelastic light scattering spectroscopy and its possible use for label-free brain tumor typing," Analytical and Bioanalytical Chemistry, vol. 409, no. 28, pp. 6613-6623, 2017.

[34] J. Gong, J. Yi, V. M. Turzhitsky, K. Muro, and X. Li, "Characterization of malignant brain tumor using elastic light scattering spectroscopy," Disease Markers, vol. 25, no. 6, pp. 303-312, 2008.

[35] H. K. Roy, Y. Liu, R. K. Wali et al., "Four-dimensional elastic light-scattering fingerprints as preneoplastic markers in the rat model of colon carcinogenesis $\tau^{2}, "$ Gastroenterology, vol. 126, no. 4, pp. 1071-1081, 2004.

[36] X. D. Li, S. A. Boppart, J. Van Dam et al., "Optical coherence tomography: advanced technology for the endoscopic imaging of Barrett's esophagus," Endoscopy, vol. 32, no. 12, pp. 921-930, 2000.

[37] Y. L. Kim, L. Yang, R. K. Wali et al., "Simultaneous measurement of angular and spectral properties of light scattering for characterization of tissue microarchitecture and its alteration in early precancer," IEEE Journal of Selected Topics in Quantum Electronics, vol. 9, no. 2, pp. 243-256, 2003.

[38] E. Ostertag, B. Boldrini, S. Luckow, and R. W. Kessler, "Labelfree multimodal microspectroscopic differentiation of glioblastoma tumor model cell lines combined with multivariate data analysis," Biophotonics: Photonic Solutions for Better Health Care III, vol. 8427, 2012.

[39] S. Nikzad, M. E. Hoenk, T. J. Jones, and S. R. Cheng, UV imaging for intraoperative tumor delineation, 2013.

[40] E. K. Ostertag, R. W. Ritz, and J.-W. Bartsch, Method for marker-free demarcation of tissues, 2015.

[41] S. A. Haider, S. Lim, S. N. Kalkanis, and I. Y. Lee, "The impact of 5-aminolevulinic acid on extent of resection in newly diagnosed high grade gliomas: a systematic review and single institutional experience," Journal of Neuro-Oncology, vol. 141, no. 3, pp. 507-515, 2019.

[42] N. Vogler, S. Heuke, T. W. Bocklitz, M. Schmitt, and J. Popp, "Multimodal imaging spectroscopy of tissue," Annual Review of Analytical Chemistry, vol. 8, no. 1, pp. 359-387, 2015.

[43] I. Georgakoudi, E. E. Sheets, M. G. Müller et al., "Trimodal spectroscopy for the detection and characterization of cervical precancers in vivo," American Journal of Obstetrics and Gynecology, vol. 186, no. 3, pp. 374-382, 2002.

[44] W. Kessler, Multivariate Data Analysis: For Pharma, Bio-and Process Analytics, John Wiley \& Sons, Hoboken, NJ, USA, 2007.

[45] T. Næs, A User-Friendly Guide to Multivariate Calibration and Classification, Vol. 6, NIR, Chichester, England, 2002.

[46] S. Guo, "Multimodal image analysis in tissue diagnostics for skin melanoma," Journal of Chemometrics, vol. 32, no. 1, Article ID e2963, 2018. 
[47] K. Zhang, C. Hao, B. Man et al., "Diagnosis of liver cancer based on tissue slice surface enhanced Raman spectroscopy and multivariate analysis," Vibrational Spectroscopy, vol. 98, pp. 82-87, 2018.

[48] P. D. Lewis, "Evaluation of FTIR spectroscopy as a diagnostic tool for lung cancer using sputum," BMC Cancer, vol. 10, no. 1, p. 640, 2010.

[49] R. Sethupathi, K. Gurushankar, and N. Krishnakumar, "Optical redox ratio differentiates early tissue transformations in DMBA-induced hamster oral carcinogenesis based on autofluorescence spectroscopy coupled with multivariate analysis," Laser Physics, vol. 26, no. 11, p. 116202, 2016.

[50] H. Abdi and L. J. Williams, "Principal component analysis," Wiley Interdisciplinary Reviews: Computational Statistics, vol. 2, no. 4, pp. 433-459, 2010.

[51] R. Bro and A. K. Smilde, "Principal component analysis," Anal. Methods, vol. 6, no. 9, pp. 2812-2831, 2014.

[52] S. Wold, K. Esbensen, and P. Geladi, "Principal component analysis," Chemometrics and Intelligent Laboratory Systems, vol. 2, no. 1-3, pp. 37-52, 1987.

[53] S. Mika, G. Ratsch, J. Weston, B. Scholkopf, and K. R. Mullers, "Fisher discriminant analysis with kernels," in Proceedings of the Neural Networks for Signal Processing IX: Proceedings of the 1999 IEEE Signal Processing Society Workshop (Cat. no. 98TH8468), Madison, WI, USA, August 1999.

[54] A. D. Meade, C. Clarke, F. Draux et al., "Studies of chemical fixation effects in human cell lines using Raman microspectroscopy," Analytical and Bioanalytical Chemistry, vol. 396, no. 5, pp. 1781-1791, 2010.

[55] Z. Huang, A McWilliams, S Lam et al., "Effect of formalin fixation on the near-infrared Raman spectroscopy of normal and cancerous human bronchial tissues," International Journal of Oncology, vol. 23, no. 3, pp. 649-655, 2003.

[56] I. A. Fiedler, M. Casanova, T. Keplinger, B. Busse, and R. Müller, "Effect of short-term formaldehyde fixation on Raman spectral parameters of bone quality," Journal of Biomedical Optics, vol. 23, no. 11, p. 116504, 2018.

[57] T. M. Pereira, M. L. Z. Dagli, G. Mennecier, and D. M. Zezell, "Influence of fixation products used in the histological processing in the FTIR spectra of lung cells," Spectroscopy: an International Journal, vol. 27, no. 5-6, pp. 399-402, 2012.

[58] M. G. Shim and B. C. Wilson, "The effects of ex vivo handling procedures on the near-infrared Raman spectra of normal mammalian tissues," Photochemistry and Photobiology, vol. 63, no. 5, pp. 662-671, 1996.

[59] J. T. Mason and T. J. O’leary, "Effects of formaldehyde fixation on protein secondary structure: a calorimetric and infrared spectroscopic investigation," Journal of Histochemistry \& Cytochemistry, vol. 39, no. 2, pp. 225-229, 1991.

[60] E. Ó. Faoláin, M. B. Hunter, J. M. Byrne et al., "Raman spectroscopic evaluation of efficacy of current paraffin wax section dewaxing agents," Journal of Histochemistry \& Cytochemistry, vol. 53, no. 1, pp. 121-129, 2005.

[61] E. O. Faolain, M. B. Hunter, J. M. Byrne et al., "A study examining the effects of tissue processing on human tissue sections using vibrational spectroscopy," Vibrational Spectroscopy, vol. 38, no. 1-2, pp. 121-127, 2005.

[62] Y. Li, L. M. Almassalha, J. E. Chandler et al., "The effects of chemical fixation on the cellular nanostructure," Experimental Cell Research, vol. 358, no. 2, pp. 253-259, 2017.

[63] F. L. J. W. Engels and F. Lottspeich, "Bioanalytik," Metabolomics, vol. 979, p. 995, 2006.

[64] L. T. Perelman, M. D. Modell, E. Vitkin, and E. B. Hanlon, "Light scattering spectroscopy: from elastic to inelastic," in
Handbook of Coherent-Domain Optical Methods: Biomedical Diagnostics, pp. 437-486, Springer, Berlin, Germany, 2013.

[65] K. Rebner, M. Schmitz, B. Boldrini, A. Kienle, D. Oelkrug, and R. W. Kessler, "Dark-field scattering microscopy for spectral characterization of polystyrene aggregates," Optics Express, vol. 18, no. 3, pp. 3116-3127, 2010.

[66] E. Ostertag, "Multimodal spatially resolved optical spectroscoy for marker-free characterization of biological materials," Dissertation thesis, University of Tübingen, Tübingen, Germany, 2014.

[67] D. Naumann, "FT-infrared and FT-Raman spectroscopy in biomedical research," Applied Spectroscopy Reviews, vol. 36, no. 2-3, pp. 239-298, 2001.

[68] K. Gajjar, L. D. Heppenstall, W. Pang et al., "Diagnostic segregation of human brain tumours using Fourier-transform infrared and/or Raman spectroscopy coupled with discriminant analysis," Analytical Methods, vol. 5, no. 1, pp. 89-102, 2013.

[69] N. Bergner, B. F. M. Romeike, R. Reichart, R. Kalff, C. Krafft, and J. Popp, "Tumor margin identification and prediction of the primary tumor from brain metastases using FTIR imaging and support vector machines," The Analyst, vol. 138, no. 14, pp. 3983-3990, 2013.

[70] R. Noreen, C.-C. Chien, M. Delugin et al., "Detection of collagens in brain tumors based on FTIR imaging and chemometrics," Analytical and Bioanalytical Chemistry, vol. 401, no. 3, pp. 845-852, 2011.

[71] K. R. Bambery, E. Schültke, B. R. Wood et al., "A Fourier transform infrared microspectroscopic imaging investigation into an animal model exhibiting glioblastoma multiforme," Biochimica et Biophysica Acta (BBA)-Biomembranes, vol. 1758, no. 7, pp. 900-907, 2006.

[72] J. F. Costello, "DNA methylation in brain development and gliomagenesis," Frontiers in Bioscience, vol. 8, no. 6, pp. s175-184, 2003. 